\title{
Crystallization in hybrid organic-inorganic materials through self-organization from 3-glycidoxypropyltrimethoxysilane
}

\author{
Plinio INNOCENZI, ${ }^{\dagger}$ Cristiana FIGUS, Tongjit KIDCHOB and Masahide TAKAHASHI* \\ D.A.P., Laboratorio di Scienza dei Materiali e Nanotecnologie, Università di Sassari and CR-INSTM, \\ Palazzo Pou Salit, Piazza Duomo 6, 07041 Alghero (Sassari), Italy \\ * Graduate School of Engineering, Osaka Prefecture University, Sakai, Osaka 5998531
}

\begin{abstract}
Hybrid organic-inorganic materials can organize into layered crystalline structures through self-organization when bridged silsesquioxanes are used in the sol-gel synthesis. Crystallization is, however, also observed in hybrid materials when 3-glycidoxypropyltrimethoxysilane is left to react in highly basic conditions; this synthesis route shows a peculiar property which is the possibility of preparing also crystalline hybrid films by controlling the sol-gel process. The crystalline films exhibit optical anisotropy and are optically transparent. In this article we briefly review the main achievements of our research group in the field.
\end{abstract}

(O2011 The Ceramic Society of Japan. All rights reserved.

Key-words : Hybrid materials, Self-organization, Hybrid crystallization

[Received February 14, 2011; Accepted March 5, 2011]

\section{Introduction}

One of the main feature of sol-gel chemistry is the possibility of preparing hybrid organic-inorganic materials through organically modified alkoxides. ${ }^{1)}$ The covalent bonding between silicon and carbon is generally quite stable in hydrolytic conditions $^{2)}$ and this property allows the synthesis, using a solgel process, of an almost endless family of hybrid materials. ${ }^{3)}$ The structure of hybrids can be extremely complex and it is quite difficult to generalize it because is very much a case by case study which has to be performed to understand in detail the intimate structure. In general, it is well understood that monofunctional alkoxides form tridimensional networks while difunctional alkoxides favour the formation of linear structures. The structure can become much more complex if the organically modified alkoxide is bearing a polymerizable function, such as an epoxy or a vinyl or a methacrylic group. In this case two networks, which are directly connected through a covalent bond, are formed: an inorganic one, from the polycondensation reaction of the silica species and an organic one from the polymerization of the organic groups. The other case is represented by hybrid materials which are obtained by bridged silsesquioxanes molecular precursors of $\left(\mathrm{R}^{\prime} \mathrm{O}\right)_{3} \operatorname{SiRSi}\left(\mathrm{OR}^{\prime}\right)_{3}$ type; ${ }^{4), 5)}$ if the nature of organic spacer, $\mathrm{R}$, and the synthesis are carefully designed it is possible to obtain a hybrid material whose structure exhibits long-range order.

The structure of hybrid materials is in general amorphous, which means that they show a disordered structure both in the organic and inorganic domains; some hybrids have also a glassy behaviour and have a glass transition temperature but very few show, however, a crystalline structure. Short range order, in general, is restricted to hybrids synthesised from the bridged silsesquioxane alkoxides that form crystalline layered structures via self-organization. This type of precursors has been also used in the synthesis of mesoporous ordered hybrid materials and hybrid crystalline layers in the pore walls have been obtained. ${ }^{\text {) }}$

\footnotetext{
Corresponding author: P. Innocenzi; E-mail: plinio@uniss.it
}

The nature of the bridging groups affects the morphology of the organized structures and helical fibers with chiral shape ${ }^{7)}$ or plate-like structures ${ }^{8)}$ can be obtained. Formation of hybrid crystals through self-organization ${ }^{9}$ ) has been obtained also by molecule-by-molecule self-assembly ${ }^{10)}$ or by specific molecular recognition through weak interactions. ${ }^{11)}$

Formation of ordered hybrid structures from alkoxides of the $\left(\mathrm{R}^{\prime} \mathrm{O}\right)_{3} \mathrm{SiR}$ type is more difficult to observe because this requires the in situ formation of organic bridging bonds between silica units or "pseudo-silsesquioxanes" species via secondary bonds. ${ }^{12)}$ The formation of the bridging units as far as selforganization are processes in competition with the kinetics of the sol-gel reactions, in particular with the hydrolysis and polycondensation of the silicon alkoxy groups. Only very specific conditions of the sol-gel chemistry allow balancing the kinetic of different processes to have self-organization. The process is even more difficult to control during deposition of films; in this case the fast evaporation of the solvent changes the kinetics of the polycondensation reactions and self-organization results more difficult to achieve. A schematic illustration of the selforganization process with $\left(\mathrm{R}^{\prime} \mathrm{O}\right)_{3} \mathrm{SiR}$ type precursor is illustrated in Fig. 1; the precursor should have a reactive functional group which is able to form an organic bridging bond. The formation in situ of a bridged silsesquioxane alkoxide in combination with the polycondensation of the silanol species favours the selforganization into layered structures.

In the present article we have reviewed the formation of organized hybrid materials which are obtained from 3-glycidoxypropyltrimethoxysilane (GPTMS), an organically modified alkoxide bearing an epoxy functions. We will briefly describe the specificity of GPTMS sol-gel chemistry to introduce immediately after the formation in situ through self-organization of hybrid layered structures in bulk and films.

\section{A short overview of the chemistry of GPTMS}

GPTMS is one of the most popular organically modified alkoxides which is widely employed for the preparation of hybrid organic-inorganic materials ${ }^{13), 14)}$ and surface modification. ${ }^{15)}$ 


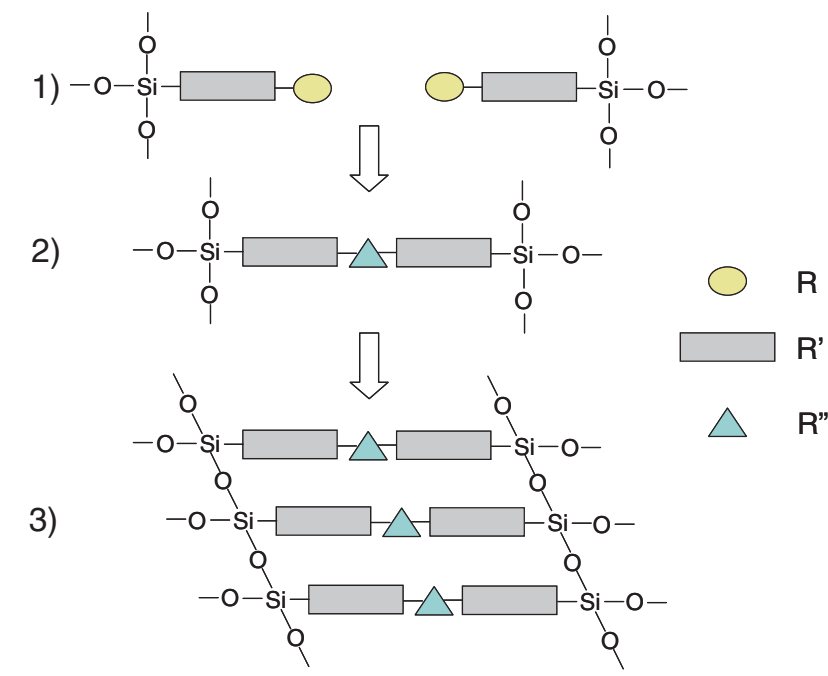

Fig. 1. (Color online) Schematic illustration of the self-organization process which involves $\left(\mathrm{R}^{\prime} \mathrm{O}\right)_{3} \mathrm{SiR}$ type precursors; the precursor has a reactive functional group which is able to form an organic bridging bond, (1). The formation in situ of a bridged silsesquioxane alkoxide in combination with the polycondensation of the silanols species (2) favours the self-organization into layered structures (3).

GPTMS has been used for different applications such as protective antiscratch coatings ${ }^{16)}$ modification of organic polymer surface ${ }^{17), 18)}$ solid electrolytes, ${ }^{19)}$ anticorrosion layers and in restoration of materials. ${ }^{20)}$ GPTMS has been also used to fabricate optical planar and channel waveguides, ${ }^{21), 22)}$ optical limiting devices, ${ }^{23)-25)}$ and for preparation of second order nonlinear optical materials. ${ }^{26)-28)}$ Several commercial materials for photonics, which are based on GPTMS are nowadays commercially available. ${ }^{29)}$ Beside this large variety of applications the chemistry of GPTMS is still showing some surprising and distinct characteristics to be used for the preparation of new types of hybrids. One of the main difficulties in controlling the chemical reactions during the sol-gel process of GPTMS is that several side reactions can be observed during the synthesis. Controlling of these reactions is never simple because little changes in the synthesis protocol can produce a strong difference in the final product.

GPTMS is characterized by the presence of an epoxy ring, whose opening allows the formation, under controlled conditions, of a poly(ethylene oxide) chain. The formation of the inorganic network, through hydrolysis and condensation reactions of the silicon alkoxy groups is simultaneously achieved with the organic polymerization. After opening of the epoxy ring, different reaction pathways and side reactions are possible. The epoxy group is capable of forming either glycol units by hydrolytic ring opening or polyethylene oxide chains (PEO) of different lengths by polymerization. The length of the organic chain is largely depending on which extent the condensation of the inorganic network has gone to completion. The formation of the organic chains and silica network is, in fact, a competitive process; ${ }^{30), 31)}$ if the polycondensation rate of the inorganic network is much faster than the polymerization of the organic side this will reduce the length of the ethylene oxide chains. This process is very much controlled by the catalyst whose concentration and nature largely affect the polycondensation reactions. ${ }^{32), 33)}$ An important role in the synthesis is also played by water and the solvent; on the other hand water and ethanol are also by-products of the GPTMS sol-gel reactions. A large variety of catalysts have been used for the opening of the epoxy groups, acid $^{16)}$ and basic ${ }^{19), 34)}$ catalysts, photocatalysts. ${ }^{35)}$ 1-methylimidazole $^{19)}$ (MI) and ( $\gamma$-aminopropyl)triethoxysilane (APTES) ${ }^{36)}$ are examples of basic catalysts, while Lewis acids, such as titanium, ${ }^{37)}$ zirconium $^{38)}$ and aluminium alkoxides ${ }^{39)}$ are the most common acid catalysts. In particular, the transition metal alkoxides are very important in the sol-gel synthesis of GPTMS hybrids because they modify the inorganic network giving rise to the formation of a mixed oxide inorganic structure. The change in composition of the oxide backbone is reflected in the material properties, such as refractive index and mechanical response. $^{40)}$

As we have previously underlined, the synthesis conditions such as $\mathrm{pH}$, composition, solvents and temperature strongly affect the final structure; upon opening of the epoxies several side reactions can be activated, the epoxy can either undergo hydrolysis or alcoholysis to form diols, terminal methyl ethers, terminal $\beta$-aminoalcohol or polyaddition reactions to form polyether chains. ${ }^{41)}$ The diols in very specific conditions of synthesis can react to form dioxane rings ${ }^{42)}$ while even breaking of the organic chain in the ether oxygen can be observed. ${ }^{43)}$ Some of the possible reactions of epoxies in GPTMS are illustrated in Fig. 2.

\section{The chemistry of GPTMS in highly basic conditions}

The chemistry of GPTMS shows some surprising possibilities in extreme conditions of $\mathrm{pH}$; at very high $\mathrm{pH}$, around 13-14, the high basic values favour a fast and extensive hydrolysis of the silicon alkoxy species while the opening of the epoxy is highly slow down. The fast hydrolysis, however, is not in general followed by full condensation of silica because the high $\mathrm{pH}$ reduces the condensation rate. These conditions can be obtained using $\mathrm{NaOH}$, which is one of the basic catalysts which are commonly employed in the synthesis of hybrids from GPTMS. If GPTMS is dissolved in an aqueous solution of $\mathrm{NaOH}$ to reach a $\mathrm{pH}>13$, the conditions that we have previously described are fulfilled; the sol can be used for deposition of films or for preparing bulk gels upon controlled aging. ${ }^{44)}$ A good description of the reactions that happen in the sol is obtained by ${ }^{13} \mathrm{CNMR}$; Fig. 3 shows the ${ }^{13} \mathrm{C}$ NMR spectra of GPTMS in a highly basic solution as a function of the aging time. The signals of epoxy decrease in intensity with aging time, while at the same time the rise of new signals due to diols, terminal methyl ether groups, polyethylene oxide and dioxane are observed. The opening of the epoxy, which in these synthesis conditions is a relatively slow process, clearly follows several reaction pathways, as shown by NMR data. The complexity of the organic chemistry of epoxy is also reflected into the inorganic chemistry of silica. In general, organically modified alkoxides of the type $\left(\mathrm{R}^{\prime} \mathrm{O}\right)_{3} \mathrm{SiR}$ show the strong tendency to form cyclic structures; several types of cages can be formed in accordance with the specific conditions of synthesis. ${ }^{29} \mathrm{Si}$ NMR analysis in solution has shown that GPTMS is fully hydrolyzed immediately after mixing with water but that with the aging of the sol several types of cyclic or even ladder like structures are formed. This is in accordance with the reaction conditions which are expected at extremely high $\mathrm{pH}$ values; cyclization of organically modified alkoxides is, in fact, a well known phenomenon that can be observed in highly acidic or basic conditions. ${ }^{45)}$ A scheme of the open silica hybrid cage formation is shown in Fig. 4; the hydrolysis of GPTMS produces silica nano-building units which are silsesquioxane-like. On the other hand the silica condensation does not go to completion 


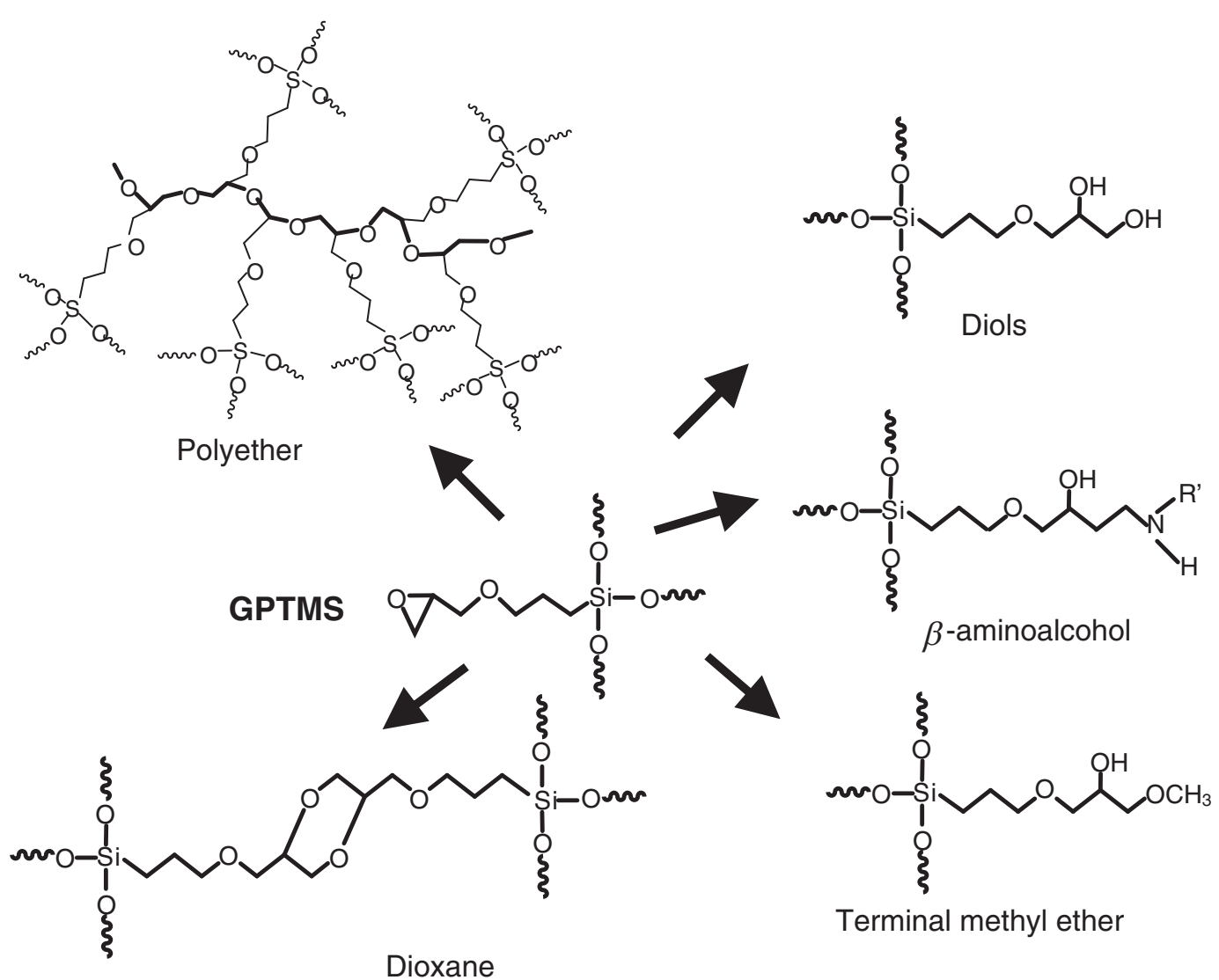

Fig. 2. Reactions pathways of epoxies in GPTMS.

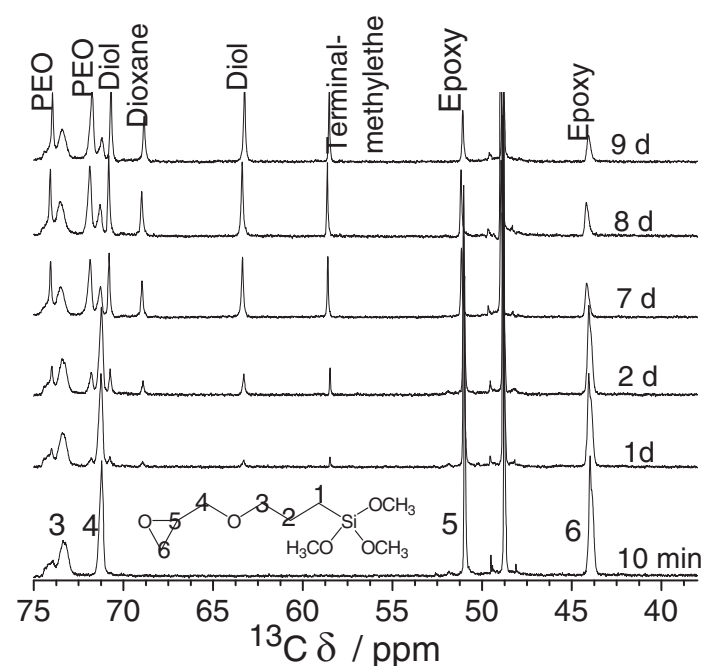

Fig. 3. ${ }^{13} \mathrm{CNMR}$ spectra of GPTMS in a highly basic solution as a function of the aging time.

even after long aging times even if a high condensation degree, up to $90 \%$, is observed after two days. If the condensation of silica reduces the free space for the growth of the polyethylene oxide chains, there is still enough space to allow forming other cross-linking bonds such as dioxanes. This reaction is extremely important because is the key for achieving in situ selforganization; when two diols in GPTMS react to form a dioxane, this results in the formation of a bridged silsesquioxane from two GPTMS molecules. The dioxane is part of the organic spacer which bridges the two silicon atoms (Fig. 5). If the hybrid

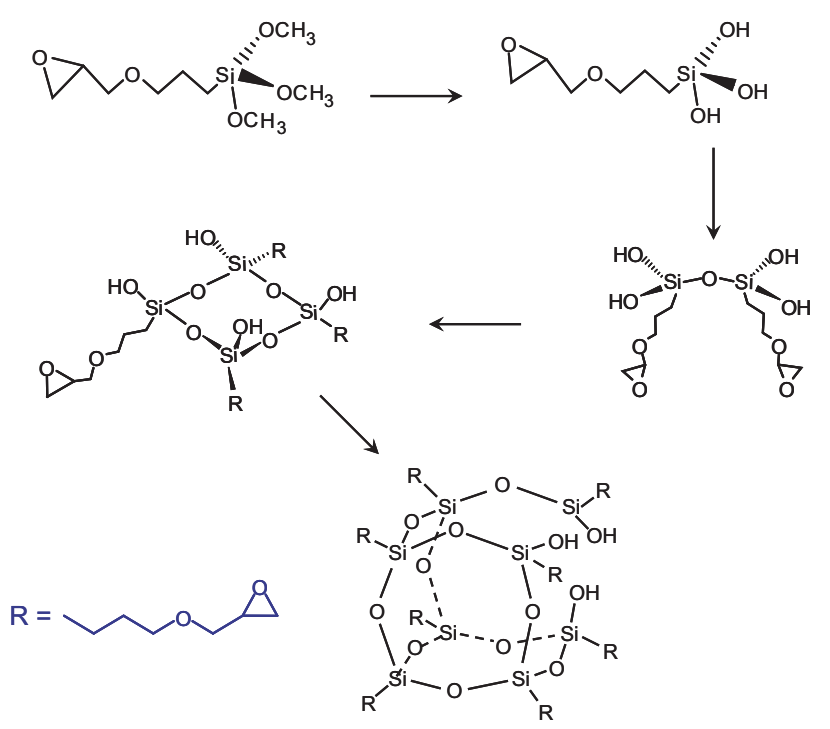

Fig. 4. (Color online) A scheme of the open silica hybrid cage formed with the hydrolysis of GPTMS; silica nano-building units which are silsesquioxane-like are produced.

network, at this point, is still enough compliant to allow structural reorganization at supramolecular level, ordered layers can be formed (Fig. 5).

\section{Self-organization in a bulk hybrid material}

The self-organization into layered ordered structures from GPTMS is a process which is controlled by the evaporation rate of the solvent which affects the polycondensation reactions. We 


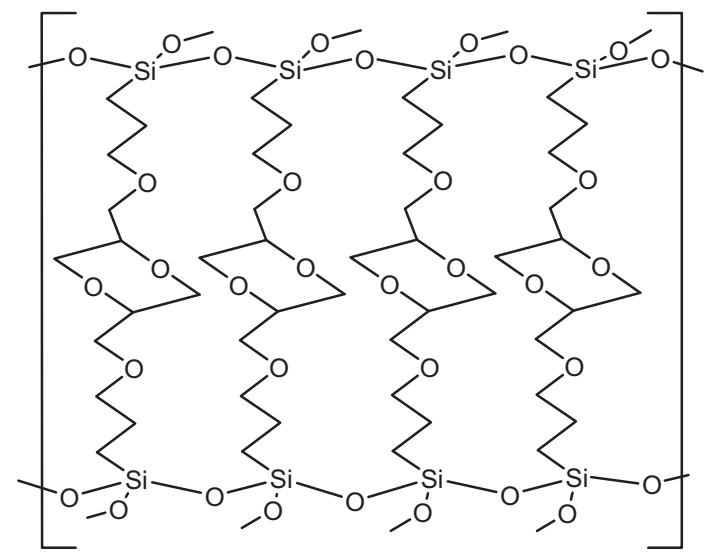

Fig. 5. A schematic illustration of the ordered hybrid structure which is obtained from reaction of GPTMS in highly basic conditions.

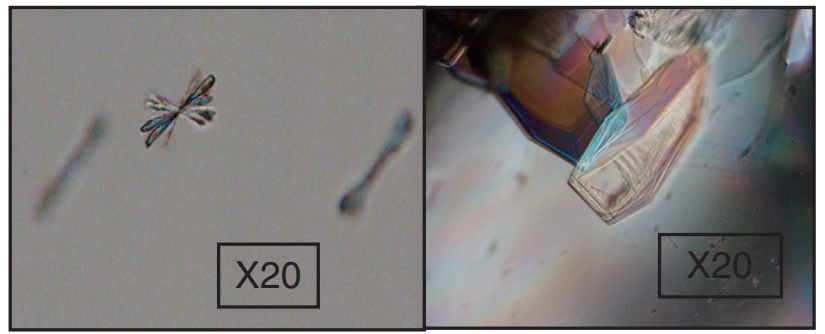

Fig. 6. (Color online) Optical images of some hybrid crystals formed in bulk.

have seen that the process is favoured by a slow condensation rate of the inorganic side and slow opening and reaction of the epoxies; on turn the matrix should remain enough compliant to allow reorganization. These conditions are relatively well fulfilled in the preparation of a bulk through the sol to gel transformation, while in films the fast evaporation of the solvent quickly changes the kinetics of the process making much more difficult the formation of hybrid crystals. Self-organization in bulks from highly basic GPTMS sols catalyzed by $\mathrm{NaOH}$ gives the formation of typical plate-like crystals which can growth up to the dimensions of several centimeters. In Fig. 6 some of the crystals obtained in the bulk are shown. The organization is kinetically controlled and crystals form only after some days of aging. The conditions of aging such as temperature, $\mathrm{RH}$ and if the aging is carried out using a closed or an open vessel, are affecting the formation of the crystals. We have followed the crystal growth in the gel phase by taking several polarized optical microscope pictures of the hybrid gel at different aging times (Fig. 6); we have observed the aggregation, then nucleation, and finally growth of hybrid crystals. When the sol is aged in an open vessel a gel layer is formed at the sol/air interface; this layer is an additional factor which facilitates the slow growth of the crystals. The evaporation of water is reduced by the presence of this gel layer and in turn adjusts the crystal growth rate so that fewer, larger crystals are formed. Analysis by SAXS and WAXS have confirmed the formation of crystalline aggregates with a layered structure. In particular the WAXS and XRD data indicate that the presence of dioxanes is directly correlated to the formation of the hybrid crystal structures. The distance of $19.51 \AA$, in fact, corresponds to the length of the organic spacer $R$ in $\mathrm{O}_{1.5} \mathrm{Si}-$ $\mathrm{RSiO}_{1.5}$ that should result from the reaction of two GPTMS $(9.75 \AA \times 2)$ via ring opening of the epoxy. A Si-substituted 2,5-bis(propoxymethyl)-1,4-dioxane is, therefore, formed.

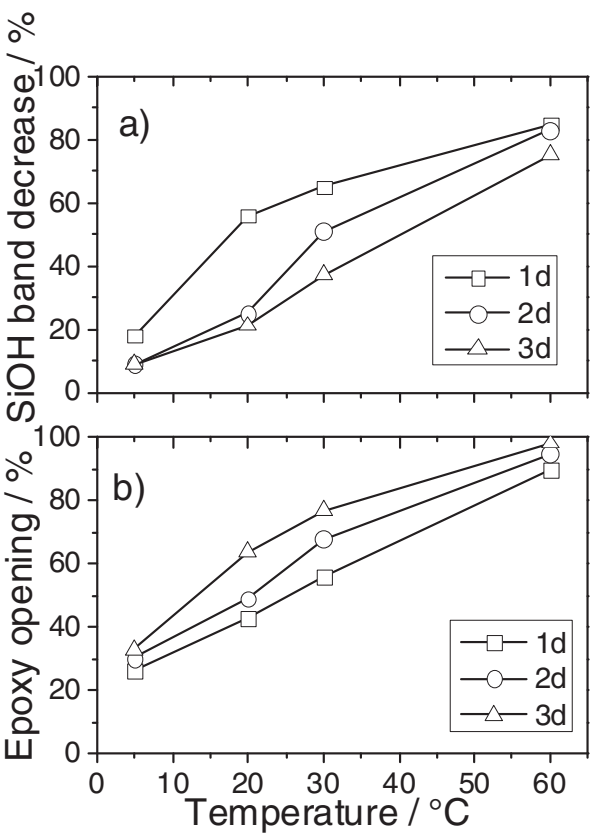

Fig. 7. a) Decrease in silanol content (variation of the $\mathrm{Si}-\mathrm{OH}$ band area at $\sim 910 \mathrm{~cm}^{-1}$ calculated with respect to the area measured in a sample prepared from a fresh sol) as a function of time and temperature of aging; 1 day (black line), 2 days (red line) and 3 days (blue line). The data have been obtained from FTIR spectra of the same samples. b) Epoxy opening (\%) as a function of time and temperature of aging; 1 day (black line), 2 days (red line) and 3 days (blue line). The data have been obtained from FT-Raman spectra of the same samples. The crosses indicate the samples that show formation of layered hybrid crystals.

\section{Self-organization in hybrid films}

Self-organization of hybrid crystals in films is more tricky because, as we have underlined, the process is kinetically controlled and if the evaporation of solvents and water is too fast it becomes extremely difficult to get organization from $\left(\mathrm{R}^{\prime} \mathrm{O}\right)_{3} \mathrm{SiR}$ type alkoxides. This difficulty can be somehow overtaken if a sol which has been aged in controlled conditions is used for the deposition of the films. In this case the crystals can nucleate in the precursor sol eventually can growth in the films. It is important that the crystals do not growth beyond the dimension of some nanometers because the films should remain optically transparent to be used in photonic applications. We have found by a systematic study of the aging ${ }^{46)}$ conditions of GPTMS precursor sols that a critical threshold of epoxy opening and silica condensation exists; the threshold represents the border between self-organization and disorder into the hybrid structures. Figure 7 shows the correlation between time and temperature of aging of the sol as a function of the epoxy content and extent of silica condensation. The change of epoxy has been calculated by Raman spectra, while silica condensation has been evapuated by FTIR spectra and expressed as relative decrease of silanol content. Self-organization is observed only in specific conditions of condensation and epoxy opening; the data show that a significant extent of silica network formation together with a high reaction rate of the epoxies are necessary conditions for observing ordered structures in the films. A specific correlation, which is represented by a critical epoxy opening and silica condensation, for crystallization of the hybrid films exists.

The formation of an ordered layered structure in the film can be assessed using gracing incidence X-ray diffraction at small 


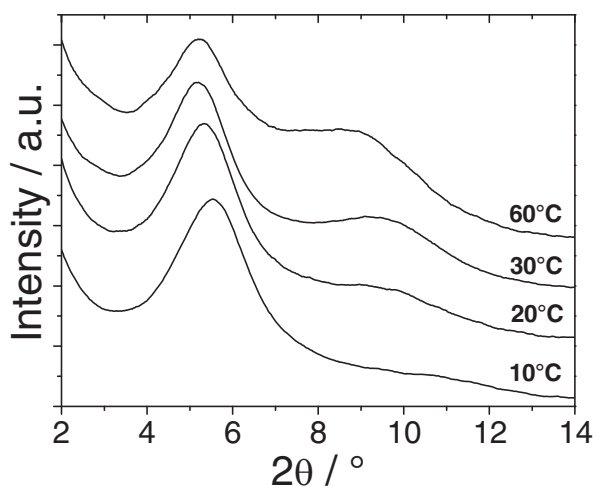

Fig. 8. XRD diffraction patterns of hybrid films prepared from a sol aged for three days at different temperatures, $10,20,30$ and $60^{\circ} \mathrm{C}$.

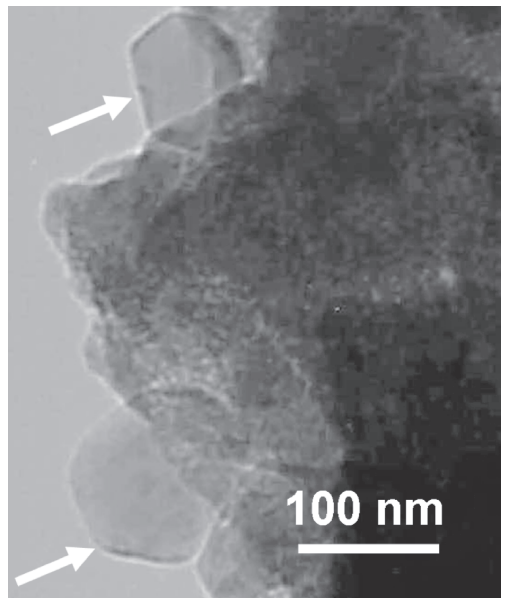

Fig. 9. TEM image of a fragment of a hybrid film containing plate like nanocrystals.

angles and direct observation by TEM combined with electronic diffraction. In Fig. 8 the XRD patterns obtained by hybrid films prepared from precursor sols aged for three days at different temperatures are shown. The different patterns are characterized by a diffraction signal peaking around $6^{\circ}$ which in general we observe to change in intensity, shape and position with the time and temperature of aging of the precursor sol. This peak, which is detected at small diffraction angles, is an indication of the presence of an intermolecular correlation and has been previously found in hybrid materials with some local order. ${ }^{47-50)}$ The formation of an ordered structure is indicated by a distinct feature in the diffraction pattern which is a broad signal of weaker intensity around $9^{\circ}$ in $2 \theta$. This signal, as observed by different authors, is the distinct fingerprint of the formation of crystalline layered organic-inorganic structures. The nature of the organized structures is directly confirmed by TEM images (Fig. 9) which show the formation of plate-like distinct structures in the nanometer scale.

The hybrid crystalline films prepared from GPTMS show a high optical transparency which is a very important property for applications in photonics. The formation of the plate like structures induce a change in the refractive index and optical anisotropy. The refractive index of the hybrid film with ordered structures have been measured to be around $1.4 \times 10^{-2}$ larger than those of amorphous hybrid film, indicating the existence of a denser crystalline phase in the material. The amorphous hybrid film has shown isotropic optical properties whilst the sample
Table 1. Refractive index, $n$, of GPTMS hybrid films which have been prepared from a fresh sol and a 15 days aged sol. The difference, $\Delta n$, in refractive index between the value obtained in TM mode and TE mode is reported in the last column on the right

\begin{tabular}{cccc}
\hline $\begin{array}{c}\text { Sol aging time } \\
\text { (days) }\end{array}$ & $\begin{array}{c}n \\
\text { (TM mode) }\end{array}$ & $\begin{array}{c}n \\
\text { (TE mode) }\end{array}$ & $\Delta n$ \\
\hline 0 & 1.48107 & 1.48090 & 0.00017 \\
15 & 1.49564 & 1.49376 & 0.00188 \\
\hline
\end{tabular}

TE mode: polarization parallel to the film surface. TM mode: polarization normal to the film surface.

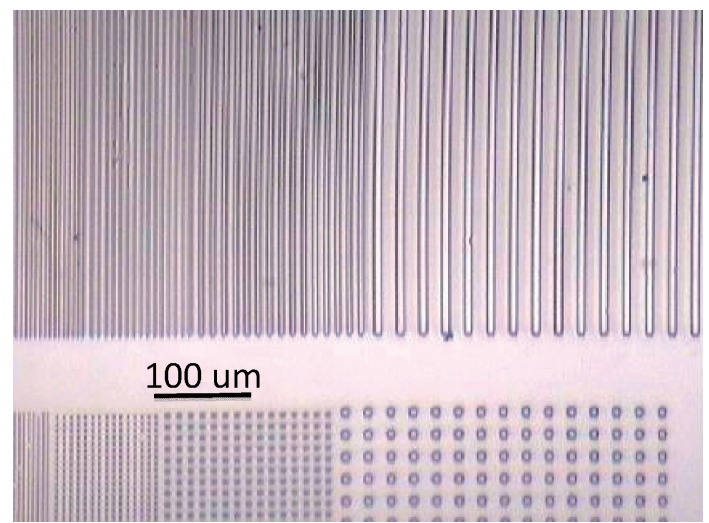

Fig. 10. (Color online) Optical image of a hybrid crystalline film patterned by soft-lithography.

with nanocrystals exhibited optical anisotropy, $\Delta n=1.88 \times$ $10^{-3}$, due to the presence of the plate shaped crystallites; the optical data are summarized in Table 1.

\section{Microfabrication}

An important property which has been demonstrated for the hybrid crystalline films is the capability of being processed for micropatterning by different techniques. We have successfully applied deep X-ray lithography and soft lithography for micro and nanopatterning of the films. In soft lithography a mold is used to write on the films different patterned structures. The films immediately after the deposition are enough viscous to be patterned but at the same time after removal of the mold they show a good capability of maintaining the pattern. An example is shown in Fig. 10 where square structures and channels with excellent spatial resolution, as high as $350 \mathrm{~nm}$, have been produced.

\section{Conclusions and a future outlook}

The formation of ordered hybrid structures in hybrid organicinorganic films has been achieved by employing a non-bridged organosilicate, 3-glycidoxypropyltrimethoxysilane. Controlling the sol-gel process allows obtaining hybrid materials which contain layered ordered domains; this route can be applied both to bulk and films. The next step is producing new families of crystalline hybrid materials with different compositions and functional properties. More studies for a better comprehension of the intimate structure of this type of hybrids are necessary; increasing the degree of crystallinity and controlling the crystals domain range is also another goal. It is also important to get a full correlation between structure and properties and to enlighten how the use of hybrids with crystalline domains has some distinctive advantages with respect to the corresponding amorphous materials. 


\section{References}

1) U. Schubert, N. Huesing and A. Lorenz, Chem. Mater, 7, 2010-2027 (1995).

2) S. Shirai, Y. Goto, N. Mizoshita, M. Ohashi, T. Tani, T. Shimada, S. Hyodo and S. Inagaki, J. Phys. Chem. A, 114, 6047-6054 (2010).

3) C. Sanchez, B. Julian, P. Belleville and M. Popall, J. Mater. Chem., 15, 3559-3592 (2005).

4) K. J. Shea and D. A. Loy, Chem. Mater., 13, 3306-3319 (2001).

5) K. J. Shea and D. A. Loy, Acc. Chem. Res., 34, 707-716 (2001).

6) S. Inagaki, S. Guan, T. Ohsuna and O. Terasaki, Nature, 416, 304-307 (2002).

7) J. J. E. Moreau, L. Vellutini, M. Wong Chi Man and C. Bied, J. Am. Chem. Soc., 123, 1509-1510 (2001).

8) J. J. E. Moreau, L. Vellutini, P. Dieudonnè, M. Wong Chi Man, J.-L. Bantignies, J.-L. Sauvajol and C. Bied, J. Mater. Chem., 15, 4943-4948 (2005)

9) F. Lerouge, G. Cerveau and R. J. P. Corriu, N. J. Chem., 30, 1364-1376 (2006).

10) S. Fujita and S. Inagaki, Chem. Mater, 20, 891-908 (2008).

11) G. Arrachart, C. Carcel, J. J. E. Moreau, G. Hartmeyer, B. Alonso, D. Massiot, G. Creff, L. Bantignies, P. Dieudonne, M. Wong Chi Man, G. Althoff, F. Babonneau and C. Bonhomme, J. Mater. Chem., 18, 392-399 (2008).

12) E. Besson, A. Mehdi, C. Reyé, P. Gaveau and R. J. P. Corriu, Dalton Trans., 39, 7534-7539 (2010).

13) M. Popall, J. Kappel, M. Pilz, J. Schulz and G. Feyder, J. Sol-Gel Sci. Technol., 2, 157-160 (1994).

14) H. Schmidt, J. Non-Cryst. Solids, 178, 302-312 (1994).

15) J. A. A. Sales and C. Airoldi, J. Non-Cryst. Solids, 330, 142149 (2003).

16) G. Philipp and H. Schmidt, J. Non-Cryst. Solids, 63, 283-292 (1984).

17) R. Nass, E. Arpac, W. Glaubitt and H. Schmidt, J. Non-Cryst. Solids, 121, 370-374 (1990).

18) J. D. Watling and D. A. Lewis, J. Sol-Gel Sci. Technol., 28, 167-174 (2003).

19) M. Popall and H. Durand, Electrochim. Acta, 37, 1593-1597 (1992).

20) P. Cardiano, S. Sergi, M. Lazzari and P. Piratino, Polymer, 43, 6635-6640 (2002)

21) P. Innocenzi, A. Martucci, M. Guglielmi, L. Armelao, G. Battaglin, S. Pelli and G. C. Righini, J. Non-Cryst. Solids, 259, 182-190 (1999).

22) G. Brusatin, M. Guglielmi, P. Innocenzi, A. Martucci, G Battaglin, S. Pelli and G. Righini, J. Non-Cryst. Solids, 220, 202-209 (1997).

23) P. Innocenzi, G. Brusatin, M. Guglielmi, R. Signorini, M. Meneghetti, R. Bozio, M. Maggini, G. Scorrano and M. Prato, J. Sol-Gel Sci. Technol., 19, 263-266 (2000).

24) P. Innocenzi, G. Brusatin, M. Guglielmi, R. Signorini, R. Bozio and M. Maggini, J. Non-Cryst. Solids, 265, 68-74 (2000).

25) R. Signorini, M. Meneghetti, R. Bozio, M. Maggini, G.
Scorrano, M. Prato, G. Brusatin, P. Innocenzi and M. Guglielmi, Carbon, 38, 1653-1662 (2000).

26) P. Innocenzi, E. Miorin, G. Brusatin, A. Abbotto, L. Beverina, G. A. Pagani, M. Casalboni, F. Sarcinelli and M. Pizzoferrato, Chem. Mater., 14, 3758-3766 (2002).

27) G. Brusatin, A. Abbotto, L. Beverina, G. A. Pagani, M Casalboni, F. Sarcinelli and P. Innocenzi, Adv. Funct. Mater. 14, 1160-1164 (2004)

28) G. Brusatin, P. Innocenzi, L. Beverina, G. A. Pagani, A. Abbotto, F. Sarcinelli and M. Casalboni, J. Non-Cryst. Solids, 345-346, 575-579 (2004).

29) B. Lebeau and P. Innocenzi, Chem. Soc. Rev., 40, 886-906 (2011).

30) P. Innocenzi, G. Brusatin, M. Guglielmi and R. Bertani, Chem Mater., 11, 1672-1679 (1999).

31) P. Innocenzi, G. Brusatin and F. Babonneau, Chem. Mater., 12, 3726-3732 (2000)

32) B. Alonso, D. Massiot, F. Babonneau, G. Brusatin, G. Della Giustina, T. Kidchob and P. Innocenzi, Chem. Mater., 17, 3172-3180 (2005)

33) P. Innocenzi, T. Kidchob and T. Yoko, J. Sol-Gel Sci. Technol., 35, 225-235 (2005).

34) B. Riegel, S. Blittersdorf, W. Kiefer, S. Hofacker, M. Muller and G. Schottner, J. Non-Cryst. Solids, 226, 76-84 (1998).

35) G. Brusatin, G. Della Giustina, M. Guglielmi and P. Innocenzi, Prog. Solid State Chem., 34, 223-229 (2006).

36) D. H. Kim, J. H. Moon, D. W. Lee and Y. G. Shul, J. Sol-Gel Sci. Technol., 26, 403-406 (2003).

37) D. Hoebbel, M. Nacken and H. Schmidt, J. Sol-Gel Sci Technol., 12, 169-179 (1998).

38) G. Philipp and J. Schmidt, J. Non-Cryst. Solids, 82, 31-36 (1986).

39) M. Templin, U. Wiesner and W. H. Spiess, Adv. Mater., 9, 814817 (1997).

40) P. Innocenzi, M. Esposto and A. Maddalena, J. Sol-Gel Sci. Technol., 20, 293-301 (2001).

41) G. Schottner, Chem. Mater., 13, 3422-3435 (2001).

42) B. Menaa, M. Takahashi, P. Innocenzi and T. Yoko, Chem. Mater., 19, 1946-1953 (2007).

43) P. Innocenzi, A. Sassi, G. Brusatin, M. Guglielmi, D. Favretto, R. Bertani, A. Venzo and F. Babonneau, Chem. Mater., 12 3635-3643 (2001)

44) P. Innocenzi, C. Figus, T. Kidchob, M. Valentini, B. Alonso and M. Takahashi, Dalton Trans., 9146-9152 (2009).

45) L. Matejka, O. Dukh, J. Brus, Jr., W. J. Simonsick and B. Meissner, J. Non-Cryst. Solids, 270, 34-47 (2000).

46) C. Figus, M. Takahashi, T. Kidchob, T. Yoko, M. Piccinini, M. Casula and P. Innocenzi, J. Sol-Gel Sci. Technol., 52, 408414 (2009).

47) B. Boury, R. J. P. Corriu, P. Delord and V. Le Strat, J. NonCryst. Solids, 265, 41-50 (2000).

48) H. B. Park, J. K. Kim, S. Y. Nam and Y. M. Lee, J. Membr. Sci., 220, 59-73 (2003).

49) H. Han and C. C. Gryte, Polymer, 63, 1663-1672 (1995).

50) C. Joly, M. Smaihi, L. Porcar and R. D. Noble, Chem. Mater., 11, 2331-2338 (1999). 